\title{
Editorial: Stability and non-linear behaviour of steel structures
}

1 Leroy Gardner BEng, MSC, PhD, DIC, CEng, MICE, MIStructE Reader in Structural Engineering, Imperial College, London, UK
2. Nuno Silvestre MSc, PhD

Associate Professor, Department of Mechanical Engineering, ICIST, IST, University of Lisbon, Lisbon, Portugal
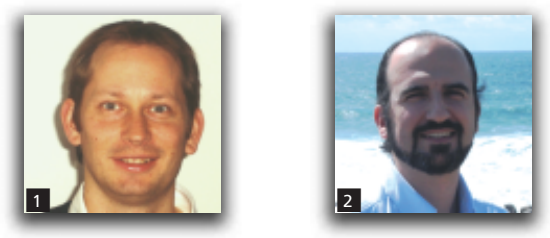

This themed issue on the Stability and Non-linear Behaviour of Steel Structures contains eight invited papers on topics presented at a Mini-Symposium of the same name convened as part of the 8th European Solid Mechanics Conference. The Conference took place in Graz, Austria from the 9th to 13th July 2012 under the auspices of EUROMECH. The papers originate from key research institutions across Europe and feature a range of instability phenomena including local, distortional and global buckling, as well as mode interaction. The studies also utilise a range of analysis techniques including mathematical modelling, physical testing and numerical simulations.

The first two papers relate to the buckling behaviour of coldformed steel lipped sections, and were prepared by Camotim and Dinis, and Casafont et al. Camotim and Dinis present a numerical study to examine the interaction of distortional and global buckling modes in both the elastic and elastic-plastic domains. Geometries were selected such that the critical loads for these two modes were almost coincident and a range of imperfections were considered. It was concluded that the reduction in ultimate strength due to mode interaction was often considerable, and that the boundary conditions of the columns (pinned or fixed) had an important effect on their behaviour. The paper by Casafont et al. concerns distortional buckling of steel storage rack columns. The primary aim of the paper is to establish a suitable standard length of member to be employed in tests on such profiles. A simple procedure, which involves a linear buckling analysis of the column, is proposed and its appropriateness is verified through a series of tests. Further investigations are ongoing.

Next, Wadee et al. present an analytical study of mode interaction in prestressed stayed steel columns, in which the addition of cross-arms and cables are used to enhance the buckling capacity of the main member. The components of the system are represented by rigid links and springs, and allow for the effects of cable slackening. The model is able to capture the general buckling response and mode interaction exhibited in previous finite element studies, and provides key insights into the response.

The fourth paper, by Taras et al., presents a numerical study of the response of steel members with mono-symmetric crosssections under compression, bending and combined loading. The results are compared with British and European design provisions, whereupon both inaccuracies and over-conservatism are uncovered. The development of improved design rules is underway.

The authors of the fifth paper, Silvestre et al., investigate the compressive behaviour of semi-elliptical hollow sections, building on recent research on fully elliptical sections, following the introduction of this shape to the range of available hot-finished hollow sections. Ultimate loads well above the critical loads of the flat portion of the section were found, due to the restraint provided by the stiffer curved portion. An expression to predict ultimate strength was also proposed.

The next two papers, by Gardner et al. and Knobloch et al., include investigations into the influence of strain-hardening on the structural response and load-bearing capacity of steel and composite sections, with the second paper considering the effects of fire. The paper by Gardner et al. makes proposals for the extension of the continuous strength method (CSM) from bare steel sections to also cover steel-concrete composite beams. The method, which was recently included in design guidance in Europe and North America for stainless steel structures, employs a deformation based approach, with allowance for strainhardening, for the determination of cross-section capacity and moves away from the traditional approach of cross-section classification. In bare steel sections, the deformation capacity is limited by local buckling or material ductility, while for composite sections, crushing of the concrete is also included. The method has been shown to provide more accurate and consistent 
prediction of cross-section capacity than traditional methods. The paper by Knobloch et al. highlights significant nonlinear material stress-strain behaviour of structural steel at elevated temperatures, particularly in the temperature region of $300-600^{\circ} \mathrm{C}$; for higher temperatures, the response is closer to the common assumption of elastic, perfectly plastic behaviour. In this scenario, the importance of a strain-based approach is emphasized, and advocated as being more suitable of fire design.

In the final paper, by Müller et al., the collapse behaviour of steel profiles is examined. In particular, an analytical method is devel- oped to predict the occurrence of a global buckling mode and a local folding mode, with the latter being favoured for energy absorption. For the cases considered, good correlation between the predictions of the analytical method and the results of numerical simulations was found.

The articles in this issue have covered a range of topics relating to the stability and nonlinear behaviour of steel structures. As well as being of great interest to researchers, this volume should be of significant relevance and interest to structural engineers engaged in the analysis and design of steel structures. 